\title{
ESTUDIO DEL MATERIAL LITICO EXCAVADO \\ EN PADRE LAS CASAS, PROVINCIA DE \\ CAUTIN. IX REGION, CHILE
}

Tom D. Dillehay - Américo Gordon

Un total de 154 objetos liticos se recolectaron en la excavación realizada en Padre Las Casils. El conjunto lítico se compone de siete unidades mayores definidos: 19) nódulos, 29) artefacios sobre nódulos, 39) lascas (monotaces), 49) bifaces, 59) martillos, 69) piedras de moler y $7^{\circ}$ ) pulidores. En casos pertinentes ada una de estas unidades líticas ha sido subdividida para definir variaciones de tipos morfológicos y determinar reducidos grupos de artefa:los, comparativamente homogéneos, los que presentan similares rasgos y posiblemonte desempeñaron análogas funciones.

A continuación se presenta la definición y descripción cle cada unidad lítica basada principalmente en Semenov, 1964; Wilmsen, 1968; Hester, 1971, y Shafer, 1971. La proceclencia de cada grupo lítico por niveles de excavación se encuentra en la Tabla Nọ 1. La cantidad de los especímenes componentes cle cada subgrupo y la naturaleza de la materia lítica de cada tipo morfológico se halla resumida en las Tablas respectivas. Se indican solamente las medidas de los artefactos tipos enteros, no fracturados (p. ej., martillos, pulidores). Algunos ejemplares de los mayores grupos morfológicos se ilustran en las Láminas 1,2 y 3.

\section{1? Nódulos. (Cantidad: 6)}

Todo guijarro o nódulo del cual una o más lascas han sido removidas se clasificó como núcleo (p. ej., Lám. 2, Fig. 3). Seis nódulos que representan el $3,9 \%$ del total de los ob- jetos líticos se hallaron en Padre Las Casas. Los nódulos incluyen 3 especímenes de basalto, I de cuarzo, 1 de cuarcita y 1 de jaspe*. Los nódulos que exhiben evidencia de adelgazamiento intencional o de utilización se clasifican separadamente.

Todos los ejemplares pueden ser clisificados como nódulos con plataforma multifacetada. El grupo incluye nódulos que antes del lascado tenían plataformas preparadas mecliante la remoción intencional de dos o más facetas para producir una plataforma de tamaño y ángulo requerido. Varios nódulos de la presente muestra parecen haber sido agotados anteriormente y luego desechados.

\section{2o Artefactos de nódulos. (Cantidad: 3)}

La clenominación "artefacto de nódulo" se refierc, en el presente estuclio, tanto a nódulos conno grandes y gruesos fragmentos de nódulos que fueron modificados intenciona!nente, con el propósito de producir artefactos funcionales para la ejccución cle trabajos de raspar, cortar o de picar. Los tres especímenes de esta categoría son labrados de basalto, representan un solo tipo morfológico y corres. ponden al $1,9 \%$ del total de la muestra lítica.

*Agradecemos a los señores profesores Ing. Arturo Arriagada C., Ing. Raúl Fonseca F., e Ing. Carlos Soto L. de la Universidad Técnica del Estado, Sede Temuco, el realizar abnegadamente el estudio petrológico. 


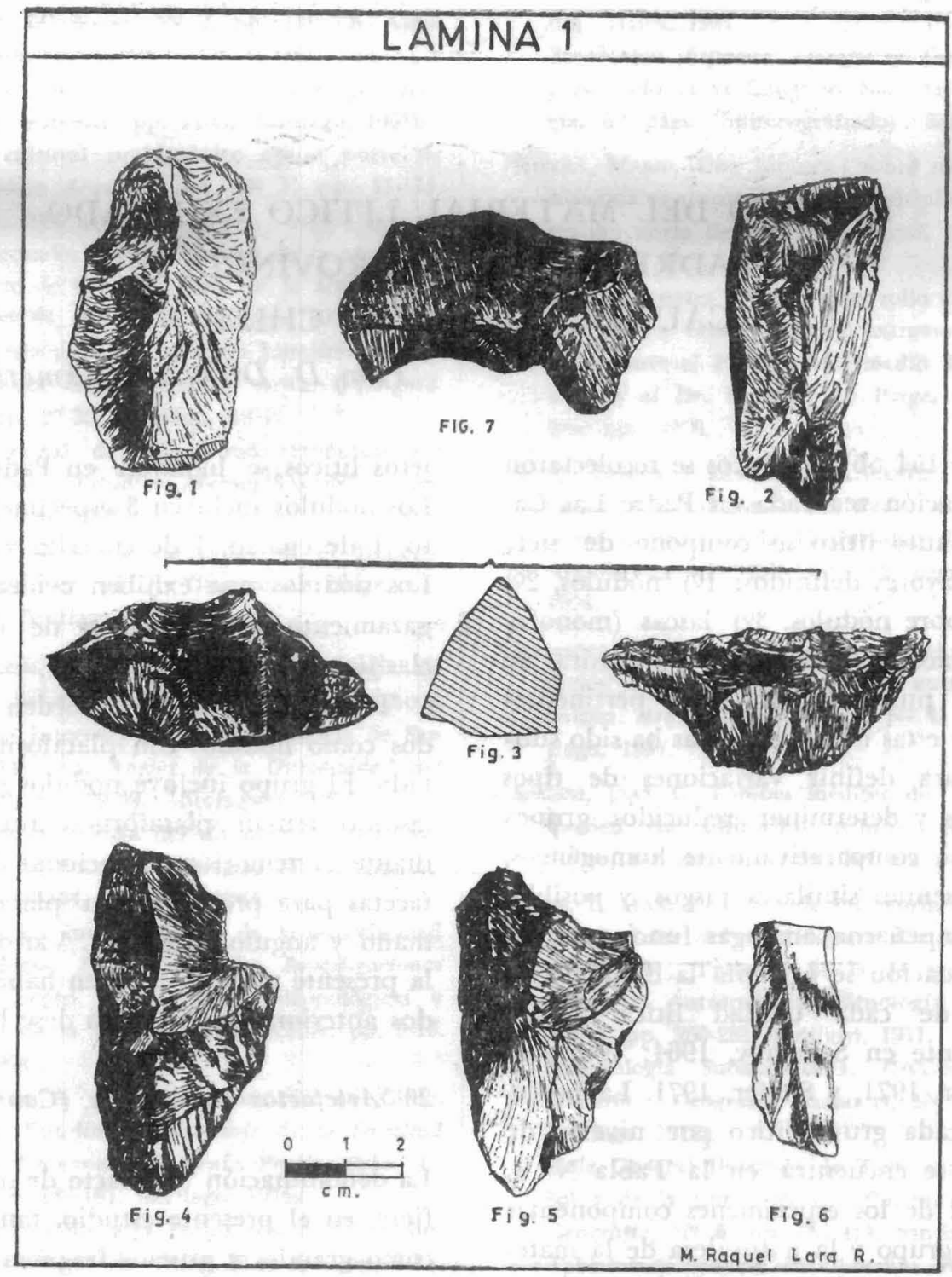

Labrados de los extremos de nódulos de forma ovalada, uno de estos objetos conserva la mayor parte de la corteza original, mientras los otros dos ejemplares solamente presentan restos del córtex. Dos piezas se caracterizan por un achaflanado agudo de lascado filudo de sus superficies. Una técnica de lascado desordenado produjo, en dos de los artefactos, márgenes sinuosos e irregulares. To. dos los ejemplares presentan una leve evidencia de uso en la forma de abrasión de sus filos como tajadores o majaderos, sugiriendo su empleo como machacadores. (Lám. 3. Fig. 1).

\section{3! Lascas. (Cantidad: 122)}

Un total de 122 artefactos, que constituyen el $79,2 \%$ de los litos excavados, se clasifican como lascas. Inicialmente las lascas se dividieron en dos subgrupos, basados en la presencia o ausencia del córtex. Estos subgrupos incluyen lascas primarias (Lám. 3. Fig. 10). Lascas secundarias (Lám. 2, Fig. 5) y terciarias (Lám. 3, Fig. 5). La subsiguiente división formó conjuntos de lascas que presentan morfológicamente similares plataformas. Los tipos de plataformas incluyen plataformas con corteza 


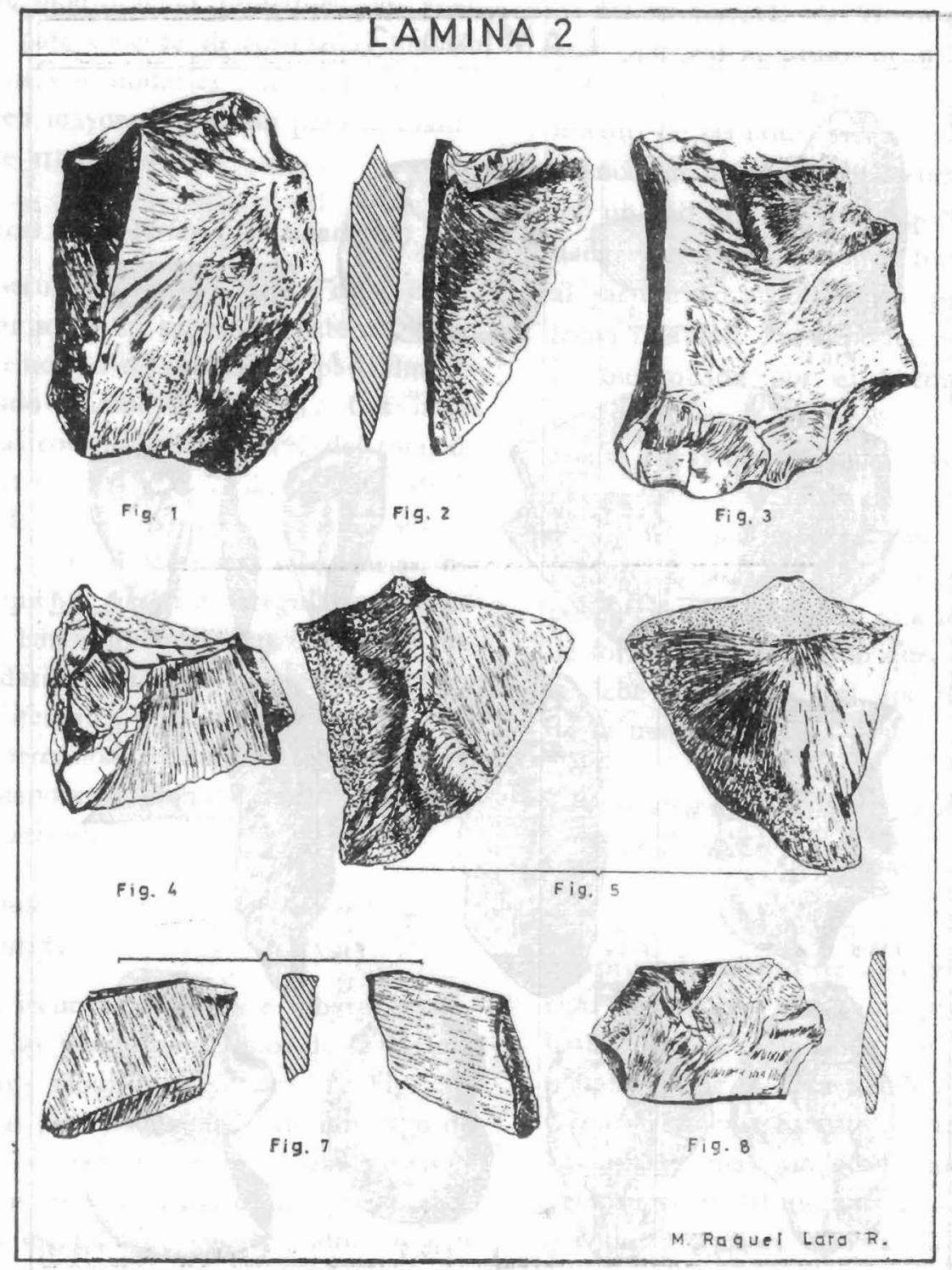

(Lám. 2, Fig. 5), plataformas monofacetadas (Lám. 1, Fig. 6) y plataformas multifacetadas (Lám. 2, Fig. 1). En el curso del proceso de clasificación todas las lascas se examinaron en busca de evidencias de modificaciones indicativas de retoques intencionales o de uso.

\section{A. Lascas primarias. (Cantidad: 11)}

Una lasca primaria se produce como resultado del ensayo inicial o del adelgazamiento marginal del nódulo. Siendo la primera lasca que se obtiene de la no-alterada superficie del nódulo, la lasca primaria ostenta gran parte de la corteza nodular, del córtex oxidado sobre toda su superficie externa. Un solo tipo de plataforma, la plataforma con corteza, es característica del desbaste de lascas primarias. Las lascas primarias constituyen el $7 \%$ de la muestra lítica excavada. Dos de estas lascas ostentan cicatrices marginales, características del proceso de modificaciones. Los once especímenes que conforman el presente grupo son de basalto.

De los dos ejemplares que demuestran evidencia de uso, uno presenta signos de empleo 


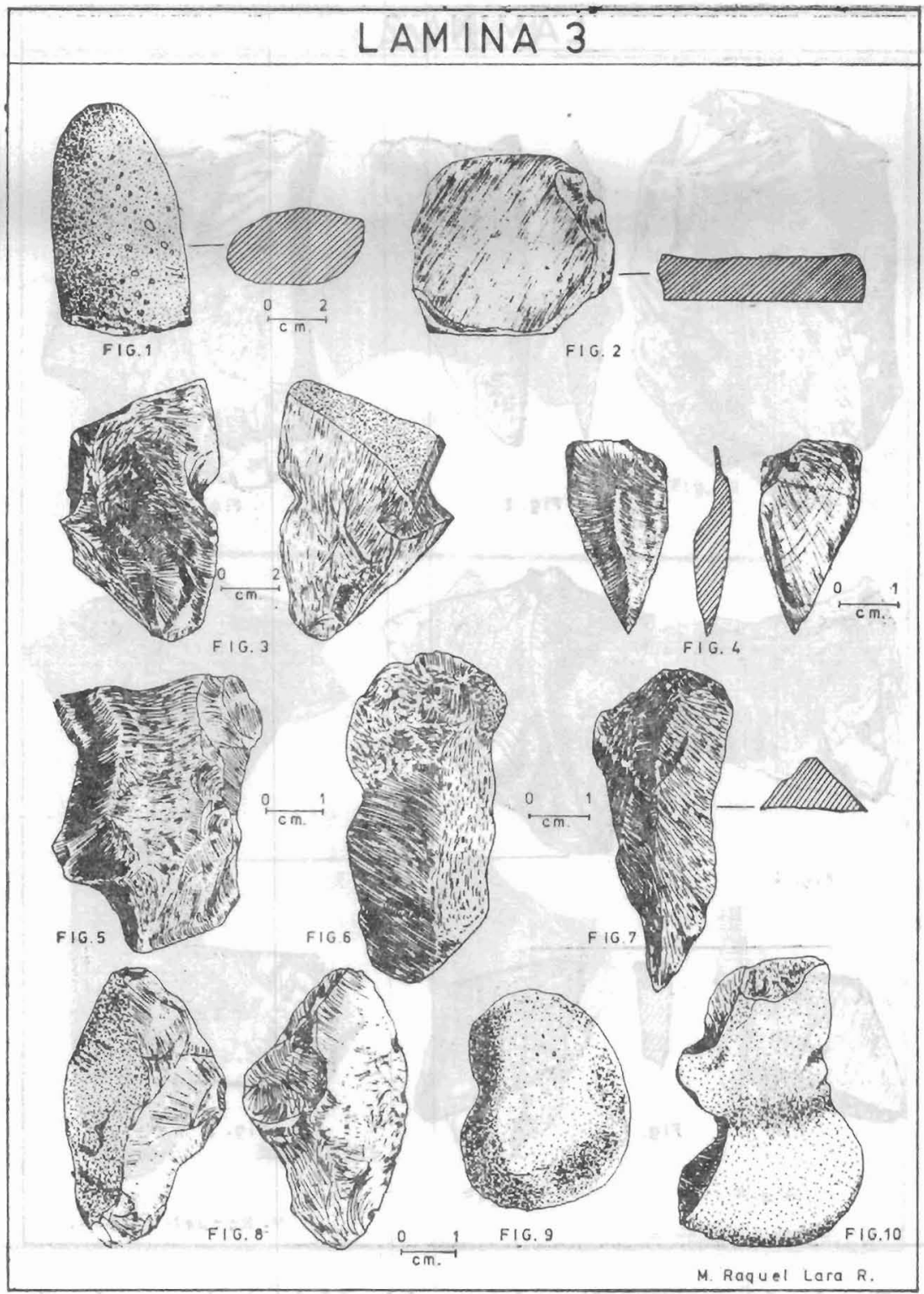

a lo largo del borde distal y el otro ha sido retocado en sus caras a lo largo de sus bordes laterales. (Lám. 3, Fig. 10).

Considerando el pequeño tamaño de los guijarros, los que se pueden obtener de las gravas del río cercano, se esperaría un porcentaje más elevado de lascas primarias de lo que se halla presente en la muestra. Aunque la cantidad total de la muestra lítica del sitio es relativamente pequeña, la aparente baja frecuencia de lascas primarias, parecería indi- car que la materia pétrea ha sido descortezada, por lo menos parcialmente, en el lugar de su recolección antes de su traslado al hábitat. La presencia en el sitio de nódulos parcialmente descortezados parece apoyar tal suposición.

Las lascas primarias tienden a ser más grandes y más gruesas que las secundarias y terciarias. La difícil tarea de remover el cór-. tex y el subsiguiente adelgazamiento de una gruesa lasca primaria, antes de su utilización 
o posterior modilicación, probablemcnte adelgazada y parcialmente descortezada, indican que las lascas secundarias y terciarias son requeridas en mayor proporción para la manulactura de artelactos.

\section{jo B. Lacas secundarias. (Cantidad: 89)}

La lasca secunclaria guarda restos de la cortezat nodular sobre su superficie externa, debido a su renoción de un nódulo parcialmente decortezado (Lán. 2. Fig. 5). Las lascals secundarias constituyen el $57,7 \%$ del total de los litos excavados y el $73 \%$ clel total de la muestra de liscas. 58 ejempiarcs, o sea, el $65.1 \%$ del total de lís lascas secundarias, ostentan pequeñas cicatrices irregulares y se supone que han siclo uzilizadas. Entre las lascas secundarias se reconocen tres, molfológicamente difarenter, lipos de plataformas o tallones de percusión: plataforma con restos de corteza, plataforma monofacetada y plataforma multifacetada.

\section{3o B-a. Plataforma con restos de corteza.}

\section{(Cantidad: 5)}

Lals lascas secundarias de este subgrupo conservan en las plataformas restos de la corteza nodular no modificada. (Lim. 2, Fig. 5). Plataformas producidas mediante este tipo de remoción tienclen a ser algo más largas y más gruesas que las plataformas preparadas. Lis lascas secundarias con plataformis con restos del córtex representan el $5,6_{\%}^{0 \%}$ del total de las lascas secundarias y todos los ejemplares son cle basalto.
En lia mayoría de los casos las lascas de este tipo son el producto de la preparación del nódulo, de la reducción o del adelgazamicnto de las lascils secundarias. La baja frecuencia de la plataforma con corteza podría ser un indicio de que pequeñas piedras rodadas y guijarros han sido trasladados del río al yacinimento arqueológico para su empleo como nódulos. I al suposición es parcialmente complubada por el hecho que las lascas con platatormas con corteza tienen generalmente pocas cicatrices dejadas por remociones previas, posible indicio de su procedencia de nódulos no expuestos previamente a un intenso lascado. Entre las lascas de este tipo de piataforma existe una amplia variedad le formas y espesores, lo que, probablemente, se debe al tamaño y al tipo de la naturaleza dic la materia pétrea.

\section{B-b. Plataforma monofacetada.}

(Cantidad: 31)

Lascas de este tipo son producidos mediante el aprovechamiento de una cicatriz de remoción dejada por una lasca previamente desbastada como plataforma de percusión. La plataforma de la lasca tiende a ser plana, lisa y completamente carente de restos del córtex. Las plataformas son generalmente, pero no siempre, bien definidas con prominentes bulbos de percusión. Las lascas monofacetadas representan el $40 \%$ del total de las lascas secundarias y veinticuatro ejemplares presentan signos de empleo.

\section{LASCAS SECUNDARIAS CON PLATAFORMA MONOFACETADA}

\begin{tabular}{|c|c|c|}
\hline Tipo de modificación & $b^{*}$ & $g^{*}$ \\
\hline lateral con muesca & 1 & 2 \\
\hline lateral distal & 7 & 1 \\
\hline lateral distal con muesca & 3 & $\mathbf{x}$ \\
\hline bilateral & 4 & $\mathbf{x}$ \\
\hline bilateral con lomo transversal & 2 & $\mathrm{x}$ \\
\hline bilateral distal & 5 & $\mathrm{x}$ \\
\hline bilateral distal con muesca & $\mathbf{I}$ & $\mathrm{x}$ \\
\hline distal & 4 & $\mathrm{x}$ \\
\hline Totales: & 27 & 3 \\
\hline
\end{tabular}

Ilustración

Lám. I. Fig. 4; Lám. 3. Fig. 3.

Lám. 1. Fig. 2; Lám. 3. Fig. 6.

Ixim. 2. Fig. 5.

Lám. 3. Fig. 8.

Lám. 1. Fig. 7 . 
La alta frecuencia de lascas con plataforma monofacetada en el presente conjunto y el correspondiente alto porcentaje de modificaciones, pueden ser indicio de la preferencia por este tipo de lascado. Este hecho, en parte, podría ser la consecuencia de la relativa facilidad con que una plataforma monofacetada puede ser preparado mediante la simple remoción de una lasca del nódulo. El pequeño tamaño de los nódulos brutos accesibles, posiblemente determinó también en el tipo de plataforma preparada para el lascado, ya que este hecho restringió, aparentemente, la producción cle largos bifaces, que es la fuente mayor de lascas multifacetadas.

La mayoría de las lascas con plataforma monofacetada conservan un alto porcentaje de la corteza nodular. Aunque las cicatrices externas tienen la tendencia de ser más numerosas en este tipo que sobre las lascas con plataforma con corteza, indicando de este modo su procedencia de nódulos o de grandes lascas, las que sufrieron un mayor grado de preparación inicial. Las lascas con plataforma monofacial parecen tener, parcialmente, un más alto porcentaje cle retoque. Si tal hecho refleja o no una preferencia cultural o si se debe simplemente a la relativa abundancia de lascas manufacturadas con plataforma monofacetada, no puede ser determi- nado a base de la presente reducida muestra.

Aclemás, es interesante observar que entre las lascas sccundarias con plataforma monofacial el astillamiento de concavidades marginales o muescas se limitó a la cara interna, aprovechando la cara externa como plataforma. A lo inverso, el retoque lateral distal marginal se limita, salvo unas pocas excepciones, a los márgenes externos, mientras los internos sirven de plataforma.

Con la excepción de dos ejemplares del grupo, las lascas aparcer: irregulares en su forma y espesor. En la inayoría de los casos la nodificación ocurre sobre el borde más largo y más delgado de las lascas.

\section{3o B-c. Plataforma multifacetada.}

\section{(Cantidad: 52)}

Plataformas de este tipo se producen me. diante la remoción intencional de dos o más largas facetas, produciendo de este modo un plano de plataforma que facilita el clesbaste de una lasca relativamente grande (Lám. 2, Figs. 4 y 8). Plataformas de grandes facetas tienden a ser más anchas y más gruesas que otros tipos de plataformas producidas. Las plataformas multifacetadas representan el $58.4 \%$ del total de la muestra de las lascas secundarias.

\begin{tabular}{|c|c|c|c|}
\hline LASCAS SE & ECUNDARIAS & CON & PL. \\
\hline Tipo de modificación & $b^{*}$ & $s *$ & $c^{*}$ \\
\hline lateral & 2 & $\mathrm{x}$ & \\
\hline lateral con lomo transversal & 4 & $x$ & $\mathrm{x}$ \\
\hline lateral distal & 7 & 1 & 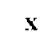 \\
\hline lateral distal con muesca & 2 & $\mathrm{x}$ & 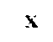 \\
\hline bilateral & 2 & $x$ & 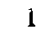 \\
\hline bilateral con muesca & 3 & $\mathrm{x}$ & $\mathbf{x}$ \\
\hline distal & $\underline{2}$ & $x$ & $\mathrm{x}$ \\
\hline Totales: & 20 & 1 & 1 \\
\hline
\end{tabular}

Las lascas con plataforma multificetada se asocian generalmente a sitios doncle se obtienen fácilmente grandes núcleos de pedernal nodular o tabular para la producción de grandes y gruesos bifaces. Las lascas con pla-

taforma multifacetada conservan solamente pequeños restos de la corteza exterior, indicando de este modo que han sido deșbastadas de núcleos intensamente labrados o de lascas secundarias de mayor tamaño. El continuado 
descortezamiento de artefactos de nódulos y el procesamiento de pequeños bifaces son probablemente la fuente principal de las Iascas con plataforma multifacetada.

\section{C. Lascas terciarias. (Cantidad: 22)}

Una lasca terciaria se caracteriza por su superficie externa que carece de corteza nodular. Las lascas terciarias proceden del interior del nódulo y son producidas después de la descortezación primaria y secundaria (Lám. 2, Figs. 2 y 7). Las lascas terciarias constituyen el $11,30^{\circ}$ ael conjunto lítico excavado y el $18 \%$ del total de la muestra de lascas y se caracterizan por plataforma monofacetada Doce ejemplares, o sea, el $54,5 \%$ de las lascas terciarias evidencian irregulares cicatrices marginales y minúsculas facetas como consecuencia die su probable empleo.

\begin{tabular}{|c|c|c|c|c|c|c|}
\hline modificación & $b^{*}$ & $c^{*}$ & $j *$ & $0^{*}$ & $m *$ & lluslración \\
\hline unilateral & 3 & 1 & 1 & $\mathbf{x}$ & $\mathbf{x}$ & Làn. 1. Fig. 6; Lám. 3. Fig. 4 \\
\hline unilateral distal & 1 & $\mathbf{x}$ & $\mathbf{x}$ & $\mathbf{x}$ & $x$ & \\
\hline bilateral & $\mathrm{x}$ & $\mathrm{x}$ & $x$ & 1 & 1 & \\
\hline bilateral distal & 1 & $x$ & $\mathrm{x}$ & 1 & $\mathbf{x}$ & \\
\hline distal & 3 & $\mathbf{x}$ & $*$ & $\mathbf{x}$ & $\mathrm{x}$ & \\
\hline Totales: & 8 & 1 & 1 & 2 & 1 & \\
\hline
\end{tabular}

Al igual que en el caso de las lascas secundarias multifacetadas, la muestra de lascas terciarias es pequeña, pero con alto por. centaje de modificaciones observables. Las lascas terciarias procedentes de la excavación de Padre Las Casas tienden a ser anchas, con el eje longitudinal más bien corto. El continuo trabajo de descortezación de grandes lascas y de bifaces es, probablemente, la fuente principal de estos productos.

\section{4o Bifaces. (Cantidad: 2)}

Un biface es un nódulo o una lasca cuyas superficies externas e internas han sido sometidas a un intenso lascado mediante percusión, descortezación y adelgazamiento. A medida que un biface se reduce y se adelgaza, con el propósito de conseguir las proporciones necesarias, el desbastador puede emplear la técnica de percusión y retoque para modelar al artefacto y producir el ángulo del filo requerido.

Los bifaces constituyen el $1.3 \%$ de la totalidad de los litos excavados. Los dos espe- címenes de basalto son gruesos e irregulares. Ambos presentan largos, aparentemente irregulares cicatrices de lascas, filos marginales convexos, sinuosos y mellados (Lám. 1, Fig. 3). Los dos bifaces tienen la forma aproximadamente ovalada.

Ambos ejemplares pueden ser denominados nódulos-bifaces, debido a la incertidumbre que existe referente a su papel en la manufactura de artefactos funcionales. Aunque podcmos suponer que los bifaces de este tipo frecuentemente se exponían a subsiguientes reducciones y modificaciones con la intención de producir un artefacto formal, pero existe otra alternativa de su probable función. Las cicarrices de lascas de los nódulos-bifaces tienden a ser grandes y delgadas, indicando su probable empleo como fuente de obtención de lascas adecuadas para modificaciones siguientes.

Sin embargo, los nódulos-bifaces presentan características que no admiten su clasificación ni como nódulos nỉ como artefactos de nódulo. Aunque los bifaces tienen una apariencia angular reminescente a nódulos in- 
tensamente trabajados, tienden a ser algo más delgados que los núcleos de nódulos y poseen bordes continuos. La ausencia de evidencia de empleo y el generalmente observado intenso lascado, que los nódulos bifaces ostentan, no permite su clasificación, en el presente caso, como artefactos de nódulos. Existe también la posibiliclacl que se trate de formas de bifaces en desarrollo, aunque su espesor, en relación a su tamaño total, no parecerá conducente al adelgazamiento controlado requerido para la manufactura de típicos bifaces.

\section{5! Piedras de moler. (Cantidad: 16)}

Once guijarros de Padre Las Casas, el 10.3\% del total de los litos, que representan evidencias de desgaste causado por el trabajo de molienda y golpeteo, se clasifican como manos de moler. Todos los especímenes son de forma ovalada y tienen caras opuestas planaj y lisas. Tres especímenes tienen cicatrices en sus puntas redondeadas, sugiriendo su empleo como machacadores también. Cinco fragmentos de manos son de basalto, tres de granito y una de toba volcánica; dos son de cuarzo, dos de olivino y uno de feldespato (Lám. 3, Fig. 1).

\section{6) Martillos. (Cantidad: 8)}

Dos guijarros, el $1.2 \%$ de la muestra lítica, presentan cicatrices causadas por golpes sobre sus cxtremos redondeados y se clasilican como piedras martillos. Uno, hecho de basalto, midle $87.4 \mathrm{~mm}$. de largo, $68.5 \mathrm{~mm}$. de ancho y $37.6 \mathrm{~mm}$. de espesor. E1 otro, de andesita, mide $116.5 \mathrm{~mm}$. de largo, $48.2 \mathrm{~mm}$. de ancho y $42.9 \mathrm{~mm}$. de grosor (Lám. 3. Fìn. 2).

\section{Pulidores. (Cantidad: 2)}

Dos guijarros de pieclra rodada presentan opuestas caras planas, alisaclas y se supone que se enuplearon como alisadores. La cicalviz de abrasión o de golpeteo en las puntas apoya tal determinación funcional. Uro de los artefactos mide $58 \mathrm{~mm}$. de largo, $29.7 \mathrm{~mm}$. de ancho y $15.1 \mathrm{~mm}$. de espesor. Este tipo de artefacto compone el $1.2 \%$ del total de los litos (Lám. 3. Fig. 9).

\section{CONSIDERACIONES FINAIES}

Tentativamente, podemos decir que el escaso material lítico excavado en Padre Las Casas, sugiere que:

19 Para la confección de artefactos líticos se emplearon principalmente guijarros y cantos rodados locales, sin embargo se recurrió también a tipos de materias pétreas que afloran en la región cordillerana, 1). ej., obsidiana. En lugares situados hacia el noroestc de Padre Las Casas se halla madera petrificada y jaspe, en abundancia;

$2^{9}$ Los pocos nódulos excavados y la ausencia de astillas, indican que el lascado de nódulos y el labrado cle lascas no se ejecutó en el sitio excavado, y

$3^{\circ}$ El predominio en la inclustria lítica de artefactos sobre lascas secundarias, sugiere su empleo en el labrado de madera o cn el procesamiento de materias vegetables.

Antes de encontramos en condiciones de enitir un resumen concluyente sobre la tecnología lítical regional, es necesario reunir más datos sobre el sitio aquí estudiado y de realizar investigaciones de campo mediante excavaciones controlaclas estratigráficamente. En vista de que gran parte de los artefactos líticos de la muestra proccle de estratos alterados, no es dada la posibilidad de observar cambios tecnológicos que han podido acontecer en el transcurso del tiempo. 


\section{GRUPOS LITICOS SEGUN NIVELES DE EXCAVACION}

\begin{tabular}{|c|c|c|c|c|c|c|c|}
\hline & 0 & 10 & 20 & 30 & 40 & 50 & \\
\hline Excavacion & - & - & - & - & - & - & \\
\hline Profundidad $\mathrm{cm}$. & 10 & 20 & 30 & 40 & 50 & 60 & \\
\hline Nivel & 1 & 2 & 3 & 4 & 5 & 6 & Total \\
\hline \multicolumn{8}{|l|}{ Grupo } \\
\hline \multicolumn{8}{|l|}{ Litico } \\
\hline I Nódulos & 1 & 2 & 0 & 2 & 1 & 0 & 6 \\
\hline \multicolumn{8}{|l|}{2 Artefactos } \\
\hline de nodulos & 0 & 1 & l & 0 & 0 & 1 & 3 \\
\hline \multicolumn{8}{|l|}{3 Luscas* } \\
\hline 3. I'rimarias & 0 & 3 & 4 & 2 & 1 & 1 & 11 \\
\hline \multicolumn{8}{|l|}{ 3B Secundarias } \\
\hline 3B-a plataforma & & & & & & & \\
\hline con córtex & 0 & 0 & 2 & 1 & 1 & 1 & 5 \\
\hline \multicolumn{8}{|l|}{ 3B-b plataforma } \\
\hline monofacetada & 2 & 2 & 18 & 11 & 2 & 2 & 32 \\
\hline \multicolumn{8}{|l|}{ 3B-c plataforma } \\
\hline multifacetada & 1 & 3 & 20 & 29 & 6 & 0 & 52 \\
\hline 3C Terciarias & 1 & 1 & 9 & 9 & 1 & l & 22 \\
\hline 4 Bifaces & 0 & 0 & 0 & 0 & 2 & 0 & 2 \\
\hline 5 Manos de moler & 0 & 9 & $\tau$ & 3 & 8 & 1 & 17 \\
\hline 6 Martillos & 0 & 1 & 1 & 0 & 0 & 0 & 2 \\
\hline 7 Pulidoras & 0 & 0 & 1 & 0 & I & 0 & 2 \\
\hline DTAL & 5 & 16 & 58 & 50 & 18 & 7 & 154 \\
\hline
\end{tabular}

* Incluidas las lascas modificadas o no modificadas.

\section{BIBLIOGRAFIA}

Hester, T. R. Bibliography of archaeology. No 1. experiments, lithic technology and petrography. Addison, Wesley Module, in Anthropology No 29. Reading, Mass.

Semenov, S. A. Prehistoric technology. (Traducido por M. W. Thompson). Cory, Adams and Mackay, London, 1964.
Sharjer, H. L. lnvestigations into South Plidins prehistory, West Central Texas. Papers of the Texas Archaeological Salvage Project. Ne 20. Iniversity of Texas, Austin, 1971 .

Wrumsen, F. W. Funcional Analysis of flaked stone artifacts. American Antiquity, Ni $33(2)$, Salt Lake City. usA 1968, pp. 156-161. 